\title{
Bilan du phosphore dans un bassin versant du lac Léman : Conséquences pour la détermination de l'origine des flux exportés
}

\section{Phosphorus mass balance and the evaluation of the origin of phosphorus outputs in a sub-watershed of lake Léman}

\author{
J. M. Dorioz, P. Quetin, J. A. Lazzarotto, J. P. Bosse et J. P. Moille
}

Volume 17, numéro 3, 2004

URI : https://id.erudit.org/iderudit/705537ar

DOI : https://doi.org/10.7202/705537ar

\section{Aller au sommaire du numéro}

\section{Éditeur(s)}

Université du Québec - INRS-Eau, Terre et Environnement (INRS-ETE)

ISSN

0992-7158 (imprimé)

1718-8598 (numérique)

\section{Découvrir la revue}

Citer cet article

Dorioz, J. M., Quetin, P., Lazzarotto, J. A., Bosse, J. P. \& Moille, J. P. (2004). Bilan du phosphore dans un bassin versant du lac Léman : Conséquences pour la détermination de l'origine des flux exportés. Revue des sciences de l'eau / Journal of Water Science, 17(3), 329-354. https://doi.org/10.7202/705537ar
Résumé de l'article

Cet article s'intéresse à la dynamique du transfert du phosphore dans un bassin rural de la région lémanique, le bassin versant du Foron $\left(51,5 \mathrm{~km}^{2}\right)$. Nous montrons qu'en établissant, pour les périodes de tarissement, la relation concentration moyenne en phosphore - débit moyen à l'exutoire, il est possible d'évaluer globalement:

1) la rétention du $P$ dans le réseau hydrographique lors de ces périodes;

2) la contribution moyenne des sources ponctuelles de $P$ aux exportations du bassin.

La rétention est un phénomène temporaire, mais c'est une étape clé de la dynamique du phosphore. Elle crée d'importants stocks de $\mathrm{P}$ dans le réseau hydrographique; ces stocks se situent pour l'essentiel au niveau des sédiments comme l'ont montré diverses études antérieures et sont exportés lors des crues. Les quantités stockées à l'issue de longs étiages dépassent souvent largement les exportations enregistrées pendant les plus fortes crues. La rétention s'accompagne d'une transformation partiellement irréversible de la spéciation du phosphore transféré.

Ces phénomènes sont pris en compte pour établir des bilans annuels de pollution, et notamment la balance diffus - ponctuel. 


\title{
Bilan du phosphore dans un bassin versant du lac Léman : conséquences pour la détermination de l'origine des flux exportés
}

\author{
Phosphorus mass balance and the evaluation of the \\ origin of phosphorus outputs in a sub-watershed of \\ lake Léman
}

J.-M. DORIOZ* , P. QUETIN, J. LAZZAROTTO, A. ORAND

Reçu le 29 janvier 2003, accepté le 12 janvier 2004**.

SUMMARY

The effective management of phosphorus in watersheds requires knowledge of the origin and magnitude of phosphorus inputs. However, for large and complex watersheds, it is impossible to determine the phosphorus mass balances by measuring the flow from each source and the rate of transfer of phosphorus originating from upstream. Because of these difficulties, we have developed a strategy based on the measurement of phosphorus mass balances during base flow periods (with no storm-flow, which means with a constant or decreasing flow). This approach was applied to the Foron River watershed (drainage area $51,5 \mathrm{~km}^{2}$, annual average water discharge $0,9 \mathrm{~m}^{3} / \mathrm{s}$ ).

The Foron River, a hillside stream with a torrential flow regime, is a tributary of Lac Léman (a large meso-eutrophic lake also known as Lake Geneva). Land use within the Foron watershed is diverse, with $50 \%$ of the watershed area being in a naturally forested area (on steep upland slopes), $36 \%$ in agricultural land lying on a gentle relief of moraine deposits $\mathbf{7 0 \%}$ permanent pasture and hay meadows, $30 \%$ cereal grain crops) and $3 \%$ in marshes. The population is scattered among four small villages, with an overall density of about 100 inhabitants per $\mathrm{km}^{2}$.

Stream flow in the river was continuously monitored and automatically sampled at the outlet from 1990 to 1993. Additional grab samples were taken at various locations, springs, agricultural sub-watersheds and brooks in the naturally forested area. Total suspended solids, soluble total phosphorus, orthophosphate and total phosphorus were determined according to standard methods. In addition, sampling was carried out to determine phosphorus stored in river sediments in places that are known to be temporary sinks for sediments.

Collaboration technique de BOSSE J.-P. et MOILLE J.-P., U.M.R. - I.N.R.A., B.P. 511, 74203 Thonon Cedex, tél. : 04502678 19, fax : 0450260760 .

* Correspondance : E-mail : dorioz@thonon.inra.fr.

** Les commentaires seront reçus jusqu'au 30 avril 2005. 
To validate the efficiency of the approach, we carried out a detailed study of the point sources and the sewage systems in the watershed. Only two-thirds of domestic wastewaters were processed in treatment plants. The minimum load of point source inputs was established to be about $67 \mathrm{~kg}$ total $P$ per week and many point sources (that is to say discharged at discrete locations) remained unidentified (i.e., not all households were connected to sewers, farmsteads contributed for unknown quantities of total $\mathbf{P}$ ).

During base flow periods, phosphorus predominantly soluble and orthophosphate, was found throughout the hydrographic network. Particulate phosphorus and suspended matter concentrations were negligible (respectively $<20 \mu \mathrm{g} / \mathrm{l}$ and $<30 \mathrm{mg} / \mathrm{l}$ ), and were not related to flow discharge and originated mainly from point source discharges. The inputs from surface runoff on the watershed were also negligible, as reflected in the low concentrations of total phosphorus in the agricultural or forested sub-watersheds (less than $15 \mu \mathrm{g} / \mathrm{L}$ ). Total inputs of phosphorus into the river included not only this background phosphorus (evaluated to be less than $2 \mathrm{~kg}$ total-P per week), but also $\mathbf{P}$ mainly originating from point sources. Total phosphorus exported from the outlet varied from 20 to $110 \mathrm{~kg}$ total-P per week but was often much lower (up to $80 \%$ ) than the minimum inputs due to point sources. During these periods phosphorus can accumulate in the hydrographic network, probably in the riverbed sediments and periphyton (macrophytes were not present).

The detailed pattern of phosphorus exports during these low flow periods showed that the amount of total phosphorus exported $(90 \%$ soluble P) tended to increase with the weekly mean water flow, up to a water discharge rate of $0.8 \mathrm{~m}^{3} / \mathrm{s}$. Above this value, phosphorus export reached a plateau corresponding to a mean constant value (about $80 \mathrm{~kg}$ per week). Since weekly average inputs from point sources remained nearly constant and independent of stream flow, this increase in $P$ export indicated an increase in the rate of transfer of total phosphorus from point sources discharged into the river. When the water discharge was $>0.8 \mathrm{~m}^{3} / \mathrm{s}$, a constant $P$ export regime was reached as all the inputs were transported to the outlet. Consequently, output at the outlet was equal to input into the river. This approach permits a calculation of the total point sources. Knowing the inputs from point sources and the total $P$ export at the outlet, we can calculate the diffuse sources.

Significant relationships between weekly mean total-P, soluble-P or orthophosphate concentrations at the outlet and weekly mean discharge were established to validate these observations. The best fit of these measured values didn't follow a standard law of dilution because the dilution effect was attenuated by the antagonist effect due to the increase in $P$ transport inputs from sewage with increasing discharge $\left([\right.$ soluble $P]=0.02+0.148 Q^{-1 / 2} ; n=82$; $\mathbf{r}^{2}=0.8$ ).

During base flow, when $Q<0.8 \mathrm{~m}^{3} / \mathrm{s}$, a portion of phosphorus inputs accumulated in the river biota and sediments through deposition, biological uptake and sorption. This accumulation may explain the higher levels of total-P and the easily exchangeable $P$ in the sediments downstream of point source discharges. The efficiency of these storage processes depends not only on water discharge, but also (secondarily) on some characteristics of the antecedent hydrological conditions. We found that the amount stored increased significantly in the weeks following large storm-flows. We assumed that this indicated the effect of an intense exchange of the $P$ loaded sediments with unsaturated sediments that were generated by erosion of natural upstream areas.

As in-stream processes alter phosphorus speciation, a part of soluble-P becomes particulate. During storm-flows following base flow periods with a discharge lower than the limit discharge, the stored phosphorus was released from both particulate and soluble forms and exported. We calculated an 
annual mass balance of soluble phosphorus to evaluate the amount of particulate phosphorus exported at the outlet due to the transformation of phosphorus from soluble to particulate forms. The result obtained suggested that 1/4 of particulate- $P(1 \mathrm{t}$ per year) resulted from this speciation change.

To summarize, analyses of phosphorus mass balances during base flow could lead to a new procedure to evaluate the mean total mass of $P$ originating from point sources, without referring to field surveys. In addition, this procedure allowed the quantification of both total-P storage as well as changes in speciation that occur during transfer of phosphorus in the hydrographic network.

Key words: phosphorus, watershed, eutrophication, sediments, point pollution, diffuse pollution.

\section{RÉSUMÉ}

Cet article s'intéresse à la dynamique du transfert du phosphore dans un bassin rural de la région lémanique, le bassin versant du Foron $\left(51,5 \mathrm{~km}^{2}\right)$. Nous montrons qu'en établissant, pour les périodes de tarissement, la relation concentration moyenne en phosphore - débit moyen à l'exutoire, il est possible d'évaluer globalement :

1) la rétention du $P$ dans le réseau hydrographique lors de ces périodes ;

2) la contribution moyenne des sources ponctuelles de $\mathbf{P}$ aux exportations du bassin.

La rétention est un phénomène temporaire, mais c'est une étape clé de la dynamique du phosphore. Elle crée d'importants stocks de $P$ dans le réseau hydrographique ; ces stocks se situent pour l'essentiel au niveau des sédiments comme l'ont montré diverses études antérieures, et sont exportés lors des crues. Les quantités stockées à l'issue de longs étiages dépassent souvent largement les exportations enregistrées pendant les plus fortes crues. La rétention s'accompagne d'une transformation, partiellement irréversible, de la spéciation du phosphore transféré.

Ces phénomènes sont pris en compte pour établir des bilans annuels de pollution, et notamment la balance diffus - ponctuel.

Mots clés : phosphore, bassin versant, eutrophisation, sédiment, pollution ponctuelle, pollution diffuse.

\section{1 - INTRODUCTION}

\subsection{Objectifs}

L'état d'un plan d'eau dépend de son bassin versant (VOLLENWEIDER, 1968). Le lac Léman, plus grand lac d'Europe occidentale, n'échappe pas à cette règle. Sous l'effet d'une charge externe excessive en phosphore $(\mathrm{P})$, le lac s'est eutrophisé. Les premiers signes de cette dégradation se sont manifestés dès les années 60 . Les mesures prises depuis lors pour contrôler cette pollution consistent, pour l'essentiel, à améliorer les réseaux d'assainissement et le traitement des eaux usées, des villes et des zones côtières. Parallèlement, en 1985, la Suisse a judicieusement interdit l'usage des tri-phosphates dans les 
lessives (BARROIN, 1992). Toutes ces actions se traduisent par une baisse des teneurs en phosphore total du Léman (CIPEL, 1991). Cependant, l'objectif affiché d'un retour à une concentration moyenne dans les eaux du lac inférieure à $0,03 \mathrm{mgP} . \mathrm{I}^{-1}$ nécessite de nouveaux efforts pour réduire les apports de $\mathrm{P}$. Toutes les origines du phosphore sont désormais concernées, y compris les sources difficiles à répertorier et à traiter du fait de leur dispersion ou de leur intermittence, comme les sources diffuses ou les rejets ponctuels d'eaux usées dans certaines zones rurales. Dans ce contexte, la maîtrise des formes du $P$ potentiellement les plus biodisponibles, c'est-à-dire les formes dissoutes et les fractions les plus labiles du phosphore particulaire (WILLIAMS et al., 1980 ; SONZOGNI et al., 1981) est essentielle.

Une telle évolution des priorités de gestion n'est pas spécifique au Léman; elle se retrouve pour la plupart des grands lacs et des écosystèmes marins côtiers des pays développés. Elle s'accompagne, pour les gestionnaires, d'un besoin de diagnostic concernant : 1) la contribution des diverses sources de $P$ à la charge externe des écosystèmes aquatiques concernés 2) les principales transformations de la spéciation du $\mathrm{P}$ qui s'opèrent lors du transfert de celui-ci dans le réseau hydrographique. Or, dans les bassins complexes et diversifiés, ces diagnostics posent de sérieux problèmes de méthode puisqu'il est impossible d'envisager des suivis simultanés et représentatifs de toutes les sources de $P$. Dans la pratique, on se contente en général de calculer les flux par des procédures qui laissent en suspens de nombreuses questions clés : charges exactes, taux et périodes de transferts, changements de spéciations...

L'objectif du travail présenté dans cet article est de contribuer à l'élaboration d'une méthode applicable à des bassins ruraux complexes pour : 1) quantifier et caractériser la dynamique de transfert d'un type de source de $P$ souvent délaissé par les scientifiques, les sources ponctuelles 2) déterminer en conséquence le "bilan de phosphore " du bassin, c'est-à-dire la contribution relative aux exportations à l'exutoire, des apports diffus, du bruit de fond naturel et des apports ponctuels 3) d'évaluer globalement l'importance des transformations $\mathrm{P}$ - dissous $\leftrightarrow \mathrm{P}$ - particulaire lors du transfert dans le réseau hydrographique.

La démarche proposée nécessite une caractérisation de la dynamique du $P$ dans le réseau hydrographique : suivi à l'exutoire des exportations à divers pas de temps, établissement de la relation concentration moyenne en $P$ - débit moyen lors des semaines de basses eaux et étude des diverses sources de $P$, dont les sédiments. Les résultats acquis permettent de discuter les priorités d'une gestion environnementale du P. Ils constituent aussi un apport aux méthodologies de suivi des bassins versants.

Cette démarche a été mise au point initialement dans un sous-bassin du Léman de $33 \mathrm{~km}^{2}$, le Redon (PILLEBouE, 1987b ; DORIOz et al., 1989) puis appliquée à l'analyse du fonctionnement et des crues du bassin voisin du Foron $\left(51,5 \mathrm{~km}^{2}\right)$ grâce à une typologie des crues (DORIOZ et al., 1998a) et enfin rendue plus opérationnelle par une étude détaillée des bilans de masse basé sur les données du suivi 1990-1993. C'est ce volet opérationnel qui fait l'objet de cet article. 


\section{2 État des connaissances}

Bien qu'il soit classique d'opposer la pollution d'origine diffuse et la pollution d'origine ponctuelle, les définitions varient selon les auteurs (NOVOTNY et OLEN, 1994). II s'ensuit une relative confusion qui, en fin de compte, rend difficile la comparaison des bilans d'une étude à une autre. Ceci nous incite à préciser, en préalable, les définitions adoptées et leurs conséquences pratiques.

Le plus cohérent et opérationnel consiste, selon nous, à classer les sources de $\mathbf{P}$ selon leurs modes d'entrée dans la rivière et leurs relations avec les conditions hydrologiques (FRERE et al., 1978). Les sources ponctuelles sont celles qui entrent dans le réseau hydrographique indépendamment de la pluviométrie, en des sites précis et relativement facilement identifiables. II s'agit très généralement de rejets à fortes concentrations en $P$, particulièrement riches en formes dissoutes. La maîtrise de cet ensemble de sources repose sur une politique de traitement des eaux usées. La mesure directe exhaustive des quantités de phosphore total entrant ponctuellement dans le réseau hydrographique n'est possible que si les eaux usées sont collectées et analysées régulièrement (KRONVANG, 1992). Cette situation est rarement rencontrée dans les bassins ruraux qui sont plutôt caractérisés par la multiplicité des points de rejets, l'absence de suivi régulier et une collecte partielle des eaux usées. Dans ce contexte les apports ponctuels au réseau hydrographique sont en général évalués par un calcul intégrant des données d'enquêtes (par exemple nombre d'habitants) et des ratios (par exemple : rejets de phosphore, en France dans les années 85-90 $\rightarrow$ 4gP/jour/habitant). Considérant ensuite que tout apport dans le réseau hydrographique est transféré à l'exutoire, on en déduit la contribution des sources ponctuelles aux quantités de $P$ exportées par le bassin. Ceci suppose implicitement que le stockage est nul à l'échelle de la période étudiée et que les sources non répertoriées sont négligeables.

Les sources diffuses présentent une dynamique bien différente : elles ne se manifestent qu'en période de ruissellement ou de ressuyage des sols. La charge en $P$ est acquise progressivement, au contact ou dans les sols agricoles, naturels ou urbains; il est difficile par conséquent, de définir un point d'entrée précis dans le réseau hydrographique. Le transfert diffus du $P$ s'accompagne de grands volumes d'eau et généralement de fortes quantités de sédiments. La maîtrise des sources diffuses de phosphore repose sur la gestion des sols, (DORIOZ et TREVISAN, 2002). Difficiles à localiser et à prévoir, les sources diffuses sont donc difficiles à quantifier (KRONVANG, 1992 ; SHARPLEY et al., 1993). De ce fait, elles sont en général évaluées par simple différence entre les exportations (flux annuels mesurés à l'exutoire) et le total des rejets ponctuels évalué sur la base d'inventaires, selon la méthode explicitée ci-dessus. Le résultat du calcul dépend étroitement de l'effort accompli pour déterminer les rejets ponctuels. II aboutit assez souvent à une surestimation du diffus, par attribution de tous les rejets ponctuels non répertoriés à des origines diffuses (PILLEBOUE, 1987b).

D'autres phénomènes peuvent également perturber le résultat de ce mode d'évaluation par différence, de la pollution diffuse. C'est en particulier le cas des rétentions du $\mathrm{P}$ dans le réseau hydrographique. Elles affectent à la fois le $\mathrm{P}$-dissous et le P-particulaire, quelles qu'en soient les origines. Le stockage s'effectue par divers processus physico-chimiques et biologiques, au niveau des sédiments, des macrophytes ou du périphyton, dans la rivière ou les zones inondables (MEALS et al., 1999; OWENS et WALLING, 2002). Actuellement, il 
n'existe pas de méthode simple permettant de connaître les quantités totales de $P$ stockées dans une rivière ou un segment de rivière. L'accroissement de la teneur en $\mathrm{P}$ des sédiments constitue seulement un révélateur du phénomène. Les études qui montrent que le phosphore ainsi retenu peut constituer une source interne significative, sont basés sur des bilans (SVENDSEN et KRONWANG, 1993 ; HARMS et al., 1978 ; PROBST, 1983). Dans le Redon et le Foron, rivières à caractère torrentiel, nous avons montré l'influence considérable de ces stockages réversibles sur les teneurs en $\mathrm{P}$ des eaux, lors des étiages comme lors de certaines crues (DORIOZ et al., 1989 ; DORIOZ et al., 1998a).

Les pertes diffuses peuvent aussi être déterminées à l'aide des « flux spécifiques ", (RYDING et RAST, 1994 ; BEHRENDT et BACHOR, 1998). Les flux spécifiques sont des valeurs de référence décrivant les pertes moyennes annuelles ( $\mathrm{kg}$ de $\mathrm{P} / \mathrm{an} / \mathrm{ha}$ ) par grands modes d'occupation des sols (cultures, herbages...). Le flux annuel diffus à l'exutoire d'un bassin, est évalué comme la somme des flux spécifiques caractéristiques de chaque parcelle du bassin considéré. Une telle démarche se heurte à deux difficultés principales.

1) Elle postule que le taux de transfert des sources diffuses au sein d'un bassin est partout de $100 \%$. Ceci est démenti par la présence, à toute échelle d'observation, de zones susceptibles de piéger le $\mathrm{P}$ émis, au moins temporairement (SVENDSEN et al., 1995 ; UUSI-KAMPA et al., 1997).

2) Elle néglige la forte variabilité inter annuelle due à l'hydrologie (JORDANMEILLE et al., 1998) et celle due aux pratiques agricoles et au sol (VANSTEELANT et al., 1997). En fait, les flux spécifiques ne devraient être utilisés que dans le cadre de comparaisons globales entre bassins (MEALS et BUDOLF, 1994), comme de simples indicateurs.

\section{2 - LE BASSIN EXPÉRIMENTAL ET SON ÉQUIPEMENT}

\subsection{Une vue d'ensemble}

Le bassin du Foron est un sous bassin du Léman situé sur sa rive sud, adjacent au bassin du Redon (figure 1). Au point de prélèvement, sa superficie est de $49 \mathrm{~km}^{2}$. II s'agit d'un bassin rural (figure 2) avec un amont pentu (pente moyenne entre 30 et $60 \%$ ) et forestier, développé sur les Préalpes calcaires (matériaux perméables) et un aval agricole sur des pentes douces $(<10 \%)$ sur des matériaux quaternaires, pour l'essentiel morainiques et peu perméables (VIAL, 1976). Les sols agricoles types sont des sols bruns profonds dont le fonctionnement hydrique est bien connu (HAMID et al., 1989).

Le Foron est une rivière à régime torrentiel avec un débit moyen annuel avoisinant $0,9 \mathrm{~m}^{3} . \mathrm{s}^{-1}$ et des eaux bicarbonatées calciques. Le caractère torrentiel limite le développement des macrophytes. L'étude des teneurs en $\mathrm{O}^{18}$ de l'eau de la rivière au niveau du point de prélèvement, montre que l'eau d'étiage provient, pour l'essentiel, de nappes situées dans la partie avale du bassin, et non pas de l'amont montagneux (A. FEHRI, Univ. Paris VI, com. pers). De la zone des villages à l'exutoire, le temps de transfert en basses eaux est assez long (par exemple, $16 \mathrm{~h}$ pour un débit proche du débit d'étiage, $\mathrm{Q}=0,2 \mathrm{~m}^{3} \cdot \mathrm{s}^{-1}$ ). 


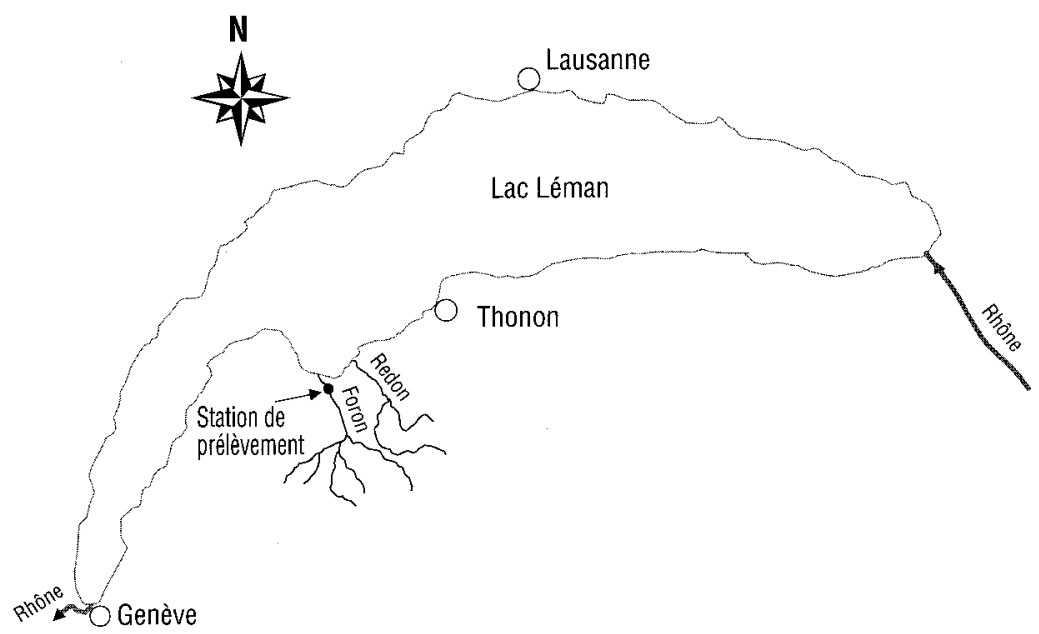

Figure 1 Carte de situation du bassin du Foron et du bassin du Léman. Location of the Foron River basin within the lake Léman area.

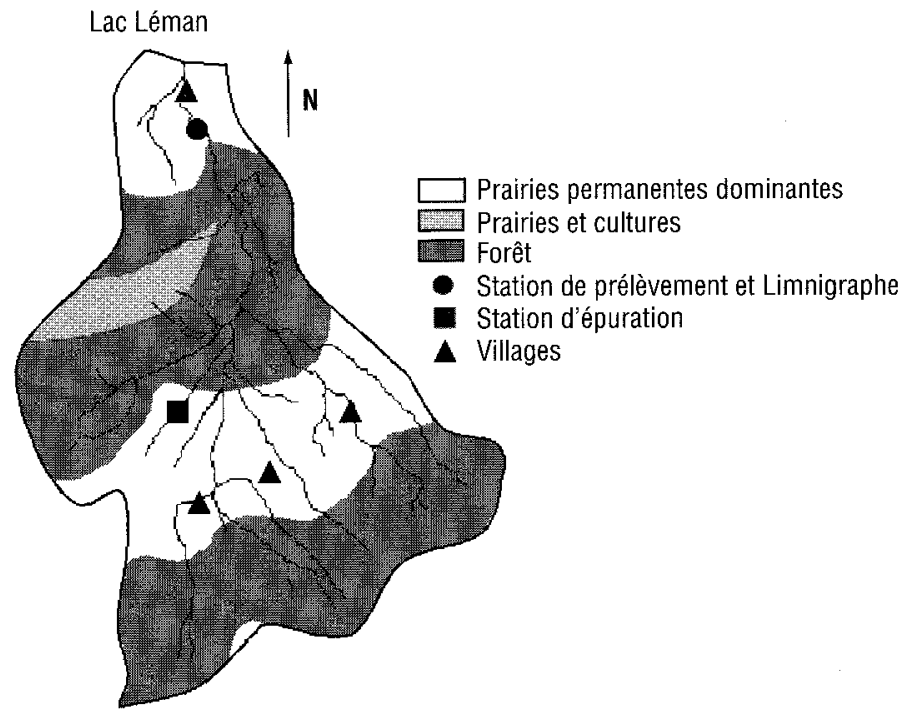

Figure 2 Mode d'occupation des sols simplifié du bassin versant du Foron.

Main characteristics of land use in the Foron River basin.

Le mode d'occupation des sols a été étudié à partir de l'image satellite SPOT de mai 1992 et d'observations de terrain (tableau 1). Les zones « naturelles » boisées représentent la moitié de la surface du bassin versant. Elles sont situées, d'une part à l'amont dans les zones en pente, et d'autre part au cceur du bassin, autour de la rivière (figure 2). L'agriculture occupe $36 \%$ du territoire avec une dominance des prairies permanentes (fauche et pâture). Les principaux systèmes d'exploitation agricoles sont des systèmes bovins laitiers relativement peu intensifs du fait de leur orientation vers une production 
fromagère de qualité (en 1992, 50 à 60 exploitations agricoles environ, soit 2000 vaches et 200 porcs). Les villages représentent une surface d'environ 50 à 60 ha (6 000 habitants en 1992).

Tableau 1 Le mode d'occupation des sols du bassin du Foron.

Table 1 Main characteristics of land use in the Foron River basin.

\begin{tabular}{lcc}
\hline & hectares & $\%$ \\
\hline Zones naturelles boisées & 2403 & 49,5 \\
Zones agricoles & 1761 & 36 \\
Zones urbaines et routes & 496 & 10 \\
Marécages & 217 & 4,5 \\
Total & 4877 & 100 \\
\hline
\end{tabular}

\section{2 Équipement et échantillonnage}

Le bassin (figure 2) est équipé, à son exutoire, d'un préleveur automatique et d'un limnigraphe relevés toutes les semaines. Les données pluviométriques utilisées sont celles de la station INRA de Thonon. Les prélèvements d'eau s'étendent de janvier 1990 à décembre 1993. En raison de la rapidité des variations de la chimie des eaux lors des montées de crue dans ce type de rivière (DORIOZ et al., 1991), les prélèvements élémentaires d'eau $(30 \mathrm{ml})$ sont réalisés toutes les 15 minutes. Les échantillons ainsi collectés sont réunis dans des flacons d'un litre correspondant à 8 heures de prélèvement. Les flacons sont en PET et prélavés à l'acide. Ils sont stockées une semaine maximum dans le préleveur, maintenus à $4{ }^{\circ} \mathrm{C}$ et à l'obscurité. Avec cette procédure, les évolutions des formes de $P$ mesurées ne sont pas significatives (DORIOZ et al., 1991).

Les analyses chimiques portent sur des échantillons moyens hebdomadaires proportionnels au débit. Le flux de chaque semaine est obtenu en multipliant la concentration moyenne de l'échantillon par le volume d'eau écoulée lors de la période considérée. À l'issue de quatre années de suivi, la banque de données utilisée pour cet article comprend l'ensemble des flux hebdomadaires dont 82 semaines de tarissement (à débit constant ou décroissant sur toute la semaine et incluant les étiages). L'étude détaillée de certaines crues est présentée dans un autre article (DORIOZ et al., 1998a). Les traitements statistiques sont effectués à l'aide du logiciel JMP (version 5, SAS Institute 2002).

En complément à ce suivi de base, quelques suivis détaillés sont réalisés pour cadrer la variabilité des périodes de tarissement (analyse de tous les flacons de $8 \mathrm{~h}$ pendant 3 semaines). Enfin, des prélèvements ponctuels d'eau sont effectués périodiquement dans le réseau principal des sources et des affluents (caractéristiques de l'amont naturel ou de sous-bassins agricoles représentatifs).

Les analyses d'eau sont réalisées selon les méthodes validées AFNOR. Elles portent sur la conductivité, le $\mathrm{pH}$, les formes du phosphore, les nitrates, les chlorures et les matières en suspension notées MES (normes : 1) conductivité à $25^{\circ} \mathrm{C}$ : NF EN 2788/ISO7888, 2) pH : NF T 90-008, 3) Orthophosphates et Phosphore total: NF EN 1189, 4) Azote nitrique : NF EN ISO 26777, 5) Chlorures : NF EN ISO 10304-1, 6) Matières en Suspension Totales : NF EN 872).

Les chlorures sont dosés en chromatographie ionique et les nitrates en colorimétrie. Dans cet article, les données nitrates et chlorure ne sont utilisées que pour des comparaisons au niveau des bilans annuels. La détermination du 
phosphore total sur eau filtrée (noté P-tef) et celle des orthophosphates (noté ortho-P) est réalisée après filtration à $0,45 \mu \mathrm{m}$. Les échantillons pour le phosphore total sur eau brute (P-total) et pour le phosphore total sur eau filtrée (P-tef) subissent une hydrolyse acide à haute température selon MENZEL et CORVIN (1965) avant une détermination spectrophotométrique selon MURPHY et RILEY (1968). Les orthophosphates sont dosés directement après filtration selon le même protocole MURPHY et RILEY (1968). Les résultats sont exprimés en phosphore élément (mg P..$\left.^{-1}\right)$. Le P-tef est considéré comme représentatif de l'ensemble des formes dissoutes. La différence P-total - P-tef constitue le $P$-particulaire (P-part). L'ensemble des méthodes d'analyse est validé bi-annuellement par des analyses inter-laboratoires.

Des sédiments sont prélevés manuellement lors des périodes d'étiage hivernal dans les tronçons de rivière à pentes faibles, au niveau de stations de la rivière où l'accumulation se fait naturellement. Les échantillons prélevés, sont tous sablo-limoneux, calcaires et oxydés. Seule la fraction dite fine $(<200 \mu \mathrm{m})$ est retenue par tamisage. L'eau interstitielle est éliminée par centrifugation à 5000 tr. $\mathrm{mn}^{-1}$. La caractérisation du phosphore des sédiments se fait selon SAUNDERS et WILLIAMS (1955) qui distinguent le phosphore total (P-total) et la fraction organique du phosphore total (noté P-orga). Une fraction labile du phosphore des sédiments est déterminée par la méthode Olsen très utilisée en agronomie (OLSEN et al., 1955) ; elle est notée P-Olsen. Les diverses teneurs en $P$ des sédiments sont exprimées en ppm ( $\mu \mathrm{g}$ de $P . \mathrm{kg}^{-1}$ de sédiment sec).

Le système d'assainissement du bassin est bien connu grâce à des parcours de terrain (repérage des rejets d'eau usées, analyses ponctuelles d'eau) et à des enquêtes auprès des responsables municipaux et d'un échantillon (10\% environ) aléatoire de la population. Nous avons également utilisé les résultats d'un travail de recensement des rejets hors normes, réalisé par l'Association de Sauvegarde du Léman.

\section{3 - ÉTAT DES LIEUX ET BILANS DES EXPORTATIONS À L'EXUTOIRE}

\subsection{Vue d'ensemble sur la variabilité des exportations à l'exutoire}

De 1990 à 1993, les pertes annuelles à l'exutoire calculées en considérant les années civiles (tableau 2) représentent en moyenne 5,4 t de P-total soit environ 8 fois moins que le flux annuel de nitrates. Le P-tef représente plus de $60 \%$ du P-total ; il est constitué à près de $90 \%$ d'ortho-P. La teneur moyenne en phosphore des suspensions varient de 300 à 1000 ppm.

Les 4 années civiles étudiées présentent un régime de température et de pluviosité peu différent de la moyenne des 20 dernières années. Les volumes écoulés varient peu et ne rendent pas compte des importantes fluctuations des flux annuels de P-part et de MES (du simple au double environ). À l'inverse, les flux annuels de $P$-tef et d'ortho-P sont relativement stables, avec des écarts à la moyenne n'excédant pas $6 \%$, ce qui est inférieur aux variations observées pour des éléments dissous et lessivables comme les nitrates (maximum $12 \%$ ) ou les chlorures (maximum $16 \%$ ). Cette stabilité relative des flux annuels de phosphore dissous a déjà été observée dans les conditions de milieu équivalentes du Redon (PILlebouE, 1987a). 
Tableau 2 Flux annuels de phosphore à l'exutoire du Foron, comparaison avec les flux de quelques autres composés et les caractéristiques hydrologiques.

Table 2 Annual $P$ fluxes at the outlet of the Foron River basin - comparison with nitrate and chloride fluxes, and with hydrological parameters.

\begin{tabular}{lccccccccc}
\hline & $\begin{array}{c}\text { P-total } \\
\text { tonnes }\end{array}$ & $\begin{array}{c}\text { P-tef } \\
\text { tonnes }\end{array}$ & $\begin{array}{c}\text { ortho } \mathbf{P} \\
\text { lonnes }\end{array}$ & $\begin{array}{c}\text { P-part } \\
\text { tonnes }\end{array}$ & $\begin{array}{c}\mathbf{N O}_{\mathbf{3}} \\
\text { tonnes }\end{array}$ & $\begin{array}{c}\text { CI } \\
\text { tonnes }\end{array}$ & $\begin{array}{c}\text { MES } \\
\text { tonnes }\end{array}$ & $\begin{array}{c}\text { Volume d'eau } \\
\mathbf{1 0}^{\mathbf{6}} \mathbf{m}^{\mathbf{3}}\end{array}$ & $\begin{array}{c}\text { Débil moyen } \\
\mathbf{m}^{\mathbf{3}} \cdot \mathbf{s}^{-\mathbf{1}}\end{array}$ \\
\hline 1990 & 4,73 & 3,50 & 3,15 & 1,23 & 34,5 & 195,3 & 1368 & 23,51 & 0,75 \\
1991 & 5,39 & 3,44 & 3,14 & 1,95 & 34,2 & 217,2 & 1777 & 21,51 & 0,68 \\
1992 & 5,19 & 3,81 & 3,39 & 1,38 & 44,2 & 262,9 & 4095 & 23,40 & 0,74 \\
1993 & 6,29 & 3,55 & 3,15 & 2,74 & 43,2 & 252,2 & 1903 & 24,90 & 0,79 \\
Moyenne & 5,40 & 3,57 & 3,21 & 1,83 & 39,0 & 231,9 & 2286 & 23,33 & 0,74 \\
\hline
\end{tabular}

Les flux hebdomadaires, varient de 20 à $500 \mathrm{~kg}$ de P-total/semaine. Une part importante des exportations du P-total se fait lors des semaines de crues. Les crues transfèrent des charges importantes, atteignant couramment $200 \mathrm{~kg} / \mathrm{jour}$ de P-total, avec une dominance (50 à $80 \%$ ) pour les formes particulaires. Hors crues, les exportations de P-total atteignent au maximum à $30 \mathrm{~kg} /$ jour et les formes dissoutes prédominent (70 à $95 \%$ ).

\subsection{Le système d'assainissement du bassin}

Les résultats sont interprétés en considérant, pour les années étudiées, un taux de rejet de phosphore total de $4 \mathrm{gP}$-total/jour/habitant (PILLEBOUE, 1987a ; BALLAND, 1989) ; ce taux diminue nettement dans les années 2000 (BOUVIER, 2003). Le taux de rétention varie de 10 à $30 \%$ du P-total dans l'unique station d'épuration (STEP) et de 10 à $20 \%$ dans le lagunage (SHELEF et al., 1983 in PILLEBOUE, 1987a). Ces valeurs moyennes sont confirmées par les mesures réalisées par les Services Techniques en charge du dossier.

L'enquête sur le système d'assainissement du bassin faite en 1990 (figure 3) montre qu'environ la moitié des rejets émis par les habitants ne rejoint pas un système de traitement. Pour une partie d'entre eux, le rejet s'effectue directement en rivière après un " assainissement " individuel sommaire et en général non entretenu. Plus de 70 tuyaux d'égouts correspondant à ce type de rejet ont été repérés tout au long du réseau hydrographique permanent, mais la quantité de P-total ainsi fournie à la rivière est difficile à quantifier. II existe aussi des puits dits "perdus " qui, en principe, étant donné la nature calcaire des terrains, ne transfèrent qu'une faible fraction du $P$ émis. La faible teneur en P-tef de l'ensemble des sources du bassin (P-tef $<0,015 \mathrm{mg} \mathrm{P.I}^{-1}$ sur 39 prélèvements) conforte cette hypothèse.

II existe également des rejets ponctuels d'eaux usées au niveau des bâtiments agricoles. En théorie, ces rejets devraient être strictement limités aux eaux de lavage des étables et des ateliers de fabrication, et traités, les autres effluents (lisier, fumier, purin) devant être stockés puis épandus comme engrais de ferme. En fait, les conditions de stockage de ces derniers notamment du purin sont rarement parfaites d'où des fuites ou des débordements et des vidanges de fosses, chroniques. La quantification de ce type de rejets ponctuels agricoles est quasiment impossible. 
Ce bref tour d'horizon confirme qu'il est difficile d'évaluer par enquête, même dans un bassin de taille moyenne comme le Foron, tous les apports au réseau hydrographique dus à la pollution ponctuelle. On peut cependant déterminer un apport minimum à la rivière de $3,5 \mathrm{t} \mathrm{P/an,} \mathrm{soit} 67 \mathrm{~kg}$ de $P$ par semaine (figure 3). Le choix d'exprimer le flux à la semaine sera justifié ultérieurement.

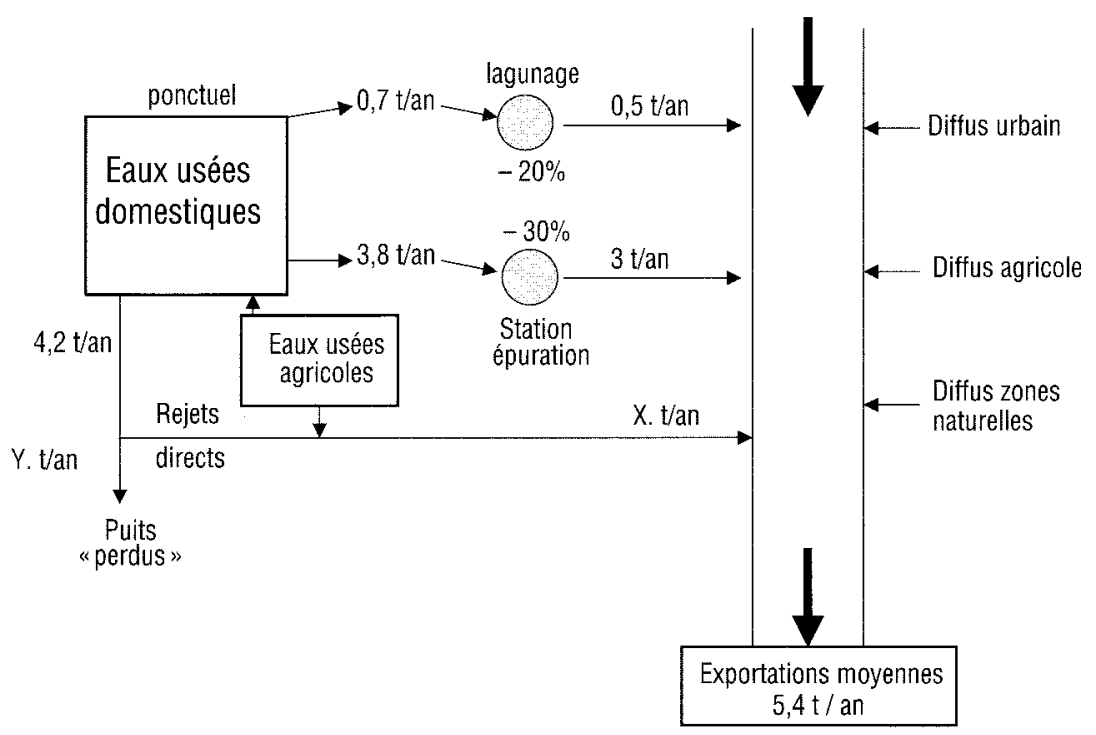

Figure 3 Le système d'assainissement et bilan du phosphore dans le bassin versant du Foron (1990-1993; les taux de rétentions \% pris en compte sont les hypothèses hautes).

Sewage system and mass balance of phosphorus in the Foron River basin (1990-1993).

\subsection{Variabilité spatiale de la pollution en étiage}

Un zonage de la rivière réalisé lors d'un étiage (5 juillet, $Q=0,1 \mathrm{~m}^{3} \cdot \mathrm{s}^{-1}$ ) souligne que seulement $10 \%$ du réseau hydrographique permanent présente une teneur en P-total de l'eau, équivalente ou proche des teneurs de l'amont forestier c'est-à-dire $<0,010$ mgP..$^{-1}$. Pratiquement, hors zone forestière, l'ensemble du réseau est donc affecté par des apports anthropiques. Dans les zones agricoles, l'accroissement des teneurs en P-tef et ortho-P des eaux se manifeste au maximum par un doublement des concentrations, alors que les rejets liés aux villages aboutissent à des concentrations beaucoup plus élevées atteignant couramment 0,4 à $0,6 \mathrm{mgP} . \mathrm{I}^{-1}$. À l'exutoire, les teneurs observées représentent 40 fois celles de l'amont " naturel " et 10 fois celles du Léman.

La même tendance à l'accroissement amont/aval de la teneur en phosphore s'observe dans les sédiments fins $(<200 \mu \mathrm{m})$ lors d'un étiage hivernal. Les concentrations sont les plus fortes dans les sédiments situés à l'aval immédiat des rejets ponctuels, avec un maximum, soit $3900 \mathrm{mgP.} \mathrm{kg}^{-1}$ sédiment, à l'aval de la station d'épuration. À l'exutoire, malgré l'éloignement des 
points de rejets $(>7 \mathrm{~km}$ ), la teneur moyenne en P-total des sédiments fins est multipliée par 2 par rapport aux sédiments fins de la zone naturelle et multipliée par 1,5 par rapport à celle d'un bassin agricole type (tableau 3). Cet accroissement de la concentration en phosphore total ne résulte pas d'un simple effet de tri granulométrique comme nous l'avons montré sur le bassin voisin du Redon (DORIOZ et al., 1989). II traduit un enrichissement de la phase solide au contact d'eaux chargées en P. II s'accompagne d'une augmentation de la proportion de P-Olsen (facilement extractible) ce qui s'exprime par l'ajustement suivant : $P$-Olsen $=0,05(P \text {-total })^{2} ;\left(P-\right.$ total en $\mathrm{mg} \cdot \mathrm{g}^{-1} ; \mathrm{R}^{2}=0,92$; $F<0,001 ; n=23$ ). À l'inverse, la teneur en $P$ organique varie sans relation significative avec le $P$ total.

Tableau 3 Comparaison de la teneur en phosphore total, phosphore organique de 4 groupes de sédiments types du bassin du Foron (sédiments $<200 \mu \mathrm{m}$; échantillons moyens récoltés lors d'un étiage prolongé ; débit moyen $=0,15 \mathrm{~m}^{3} \cdot \mathrm{s}^{-1}$ ).

Table 3 Total- $P$ and organic- $P$ content of sediments collected in 4 typical locations in the Foron River basin (sediment $<200 \mu \mathrm{m}$; low flow period samples, $Q=0.15 \mathrm{~m}^{3} \cdot \mathrm{s}^{-1}$ ).

\begin{tabular}{|c|c|c|c|c|}
\hline & $\begin{array}{l}\text { Sédiments } \\
\text { de l'amont } \\
\text { (non pollué) }\end{array}$ & $\begin{array}{c}\text { Sédiments } \\
\text { à l'aval } \\
\text { des rejets } \\
\text { (villages, step) }\end{array}$ & $\begin{array}{c}\text { Sédiments } \\
\text { d'un sous bassin } \\
\text { agricole }\end{array}$ & $\begin{array}{l}\text { Sédiments } \\
\text { de l'exutoire }\end{array}$ \\
\hline Nombre d'échantillons & 5 & 3 & 7 & 8 \\
\hline $\begin{array}{c}\text { P-total } \\
\text { mgP. } \mathrm{kg}^{-1} \text { sédiment }\end{array}$ & $460-560$ & $1080-3920$ & $560-700$ & $930-1100$ \\
\hline $\begin{array}{c}\text { P-orga } \\
\text { mgP. } \mathrm{kg}^{-1} \text { sédiment }\end{array}$ & $135-157$ & $320-400$ & $200-280$ & $260-330$ \\
\hline $\begin{array}{c}\mathrm{P}-\mathrm{Olsen} \\
\mathrm{mgP} \cdot \mathrm{kg}^{-1} \text { sédiment }\end{array}$ & $7-12$ & $21-62$ & $10-15$ & $16-21$ \\
\hline
\end{tabular}

\section{4 - BILAN EN PÉRIODE DE TARISSEMENT}

\subsection{Vue d'ensemble des teneurs dans le réseau hydrographique}

Le tarissement est défini comme la période qui suit la décrue, période pendant laquelle le ruissellement de surface est nul, l'écoulement hypodermique réduit, le débit constant ou décroissant (CASTANY, 1962). Les prélèvements ponctuels réalisés à l'exutoire lors de ces périodes (débits de 0,08 à 1,2 $\mathrm{m}^{3} . \mathrm{s}^{-1}$ ) montrent des concentrations moyennes en $\mathrm{P}$-total relativement élevées (voisines

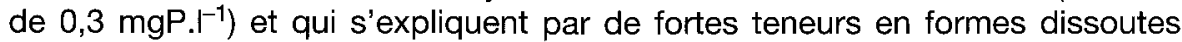

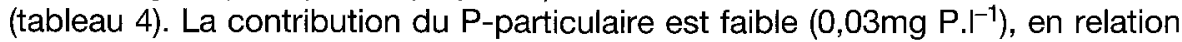
le niveau peu élevé des MES mesurées simultanément (en général $<40 \mathrm{mg}^{-1}{ }^{-1}$ ).

L'eau prélevée pendant les mêmes périodes dans les sources et les ruisseaux de sous-bassins représentatifs des zones agricoles et naturelles inhabitées de la région étudiée, est toujours 10 à 20 fois moins concentrée en 


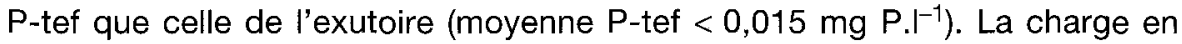
$P$-tef à l'exutoire lors des tarissements, résulte surtout donc des rejets d'eaux usées, seule source de $\mathrm{P}$ active à ce moment et présentant une forte concentration. Une estimation simpliste par calcul de la concentration moyenne de P-total minimum due aux rejets ponctuels $\left(0,22 \mathrm{mg} \cdot \mathrm{P} . \mathrm{I}^{-1}\right)$ confirme cette observation.

Tableau 4 Comparaison de la spéciation et des concentrations moyennes en phosphore en périodes de tarissement, selon l'origine des eaux, dans le bassin du Foron et dans des bassins types adjacents ( 1 : Dorioz et Ferhi, 1994 ; 2 : Pilleboue et Dorioz, 1987; moyennes arithmétiques).

Table 4 Speciation and concentration of phosphorus in water at different locations within the hydrographic network of the Foron River. (1: Dorioz et Ferhi, 1994; 2: Pilleboue et Dorioz, 1987).

\begin{tabular}{|c|c|c|c|c|}
\hline & $\begin{array}{c}\text { Nombre } \\
\text { de données }\end{array}$ & $\begin{array}{l}\text { P-total } \\
\text { mg P.I-1 }\end{array}$ & $\begin{array}{c}\text { P-tef } \\
\text { mg P.I }\end{array}$ & $\begin{array}{l}\text { Ortho-P } \\
\text { mg P. } I^{-1}\end{array}$ \\
\hline $\begin{array}{l}\text { Foron } \\
\text { exutoire }\end{array}$ & 60 & 0,315 & 0,285 & 0,183 \\
\hline $\begin{array}{l}\text { Bassin agricole type } \\
\text { (1) }\end{array}$ & 50 & 0,020 & 0,015 & 0,010 \\
\hline Zones naturelles du Foron et sources & 35 & 0,015 & 0,010 & 0,008 \\
\hline $\begin{array}{c}\text { Rejets d'égouts domestiques } \\
\text { (2) }\end{array}$ & 30 & $\begin{array}{l}\text { très variable de } 1 \\
\text { à } 30 \text { mg. } .^{-1} \\
\text { (selon dilution) }\end{array}$ & $80 \%$ & $50 \%$ \\
\hline $\begin{array}{l}\text { Estimation concentration } \\
\text { théorique due aux rejets ponctuels }\end{array}$ & - & $>0,220$ & \multicolumn{2}{|c|}{$\begin{array}{l}\text { mode de calcul : flux ponctue } \\
\text { hebdomadaire minimum } \\
(67 \mathrm{~kg} \text { de } \mathrm{P} \text { total), divisé } \\
\text { par le volume moyen } \\
\text { maximum en tarissement }\end{array}$} \\
\hline
\end{tabular}

\subsection{Variabilité des flux à l'exutoire et pas de temps minimum des bilans}

Les rejets d'eaux usées, phénomène bien connu des gestionnaires de station d'épuration, s'effectuent de façon plus ou moins cyclique. Ceci se traduit, à l'exutoire du Foron, par des variations régulières des teneurs et des flux que révèlent le suivi détaillé d'un tarissement hivernal (comprenant une première semaine avec une légère baisse du débit de 0,28 à $0,22 \mathrm{~m}^{3} \cdot \mathrm{s}^{-1}$, puis une stabilisation à $0,22 \mathrm{~m}^{3} \cdot \mathrm{s}^{-1}$ pendant 3 semaines).

Pendant ce suivi, le P-tef, qui constitue de 90 à $95 \%$ du P-total, fluctue alors que les nitrates et les chlorures présentent des teneurs quasiment constantes (respectivement : $2,4 \mathrm{mg} . \mathrm{N} / \mathrm{l}$ et $14,5 \mathrm{mg} / \mathrm{l}$ ). Le cycle journalier des rejets se manifeste à l'exutoire par des concentrations et des flux de P-tef systématiquement plus faibles (de 10 à $30 \%$ ) pour les échantillons moyens récoltés entre minuit et $8 \mathrm{~h}$ du matin (figure 4). Cette différence, sur la gamme observée, n'est liée ni à un jour particulier de la semaine, ni au débit.

À l'échelle hebdomadaire, les flux sur $8 \mathrm{~h}$ sont maximums en milieu de semaine (figure 4, flacons 7 à 17), mais les fluctuations se compensent puisque les flux hebdomadaires mesurés sont stables (dans une fourchette de $10 \%$ ) 
une fois le débit lui même stabilisé (à $0,22 \mathrm{~m}^{3} \cdot \mathrm{s}^{-1}$ ). Cette stabilité des flux hebdomadaires à l'exutoire se confirme si l'on considère l'ensemble des valeurs disponibles dans la même gamme de débit.

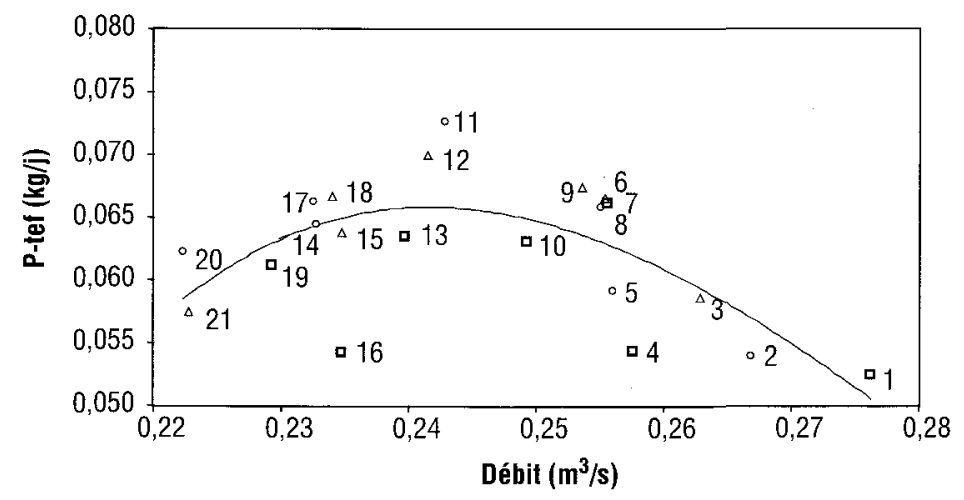

Heure de prélèvement : a de 0 à $8 \mathrm{~h} \quad \circ$ de 8 à $16 \mathrm{~h} \quad \Delta$ de 16 à $24 \mathrm{~h}$

Figure 4 Variabilité des flux selon l'heure et le jour de prélèvement pendant une semaine de tarissement (du 8 au 15/02/1993; chaque analyse correspond à $8 \mathrm{~h}$ de prélèvements intégrés; les numéros se réfèrent à l'ordre chronologique des échantillons).

Temporal variability of $P$ fluxes during a winter low flow period (February 1993).

Tous ces éléments permettent de considérer que la semaine, comme nous avions déjà pu le montrer pour le Redon (PILLEBOUE, 1987a), est le pas de temps nécessaire pour que le prélèvement à l'exutoire intègre de variabilité cyclique des rejets ponctuels. De ce fait, la semaine est aussi le pas de temps minimum pour raisonner des bilans de $P$ à rejets ponctuels en moyenne constants.

\subsection{Effet du débit sur les flux hebdomadaires exportés à l'exutoire}

Sur toute la période d'étude, les débits moyens hebdomadaires varient, en tarissement de $0,05 \mathrm{~m}^{3} \cdot \mathrm{s}^{-1}$ à $1,2 \mathrm{~m}^{3} \cdot \mathrm{s}^{-1}$. Notre série de données s'arrête à $1,2 \mathrm{~m}^{3} \cdot \mathrm{s}^{-1}$ car, pour des valeurs de débit supérieures, il n'existe plus de période de tarissement d'au moins une semaine. Dans ce cadre, les semaines de tarissement se caractérisent par une diminution de la concentration moyenne hebdomadaire en $\mathrm{P}$-total, $\mathrm{P}$-tef et ortho- $\mathrm{P}$, proportionnelle à l'accroissement du débit moyen hebdomadaire (figure 5a).

Cette évolution des teneurs en $\mathrm{P}$ est d'abord associée à la dilution d'un apport constant, celui des rejets ponctuels hebdomadaires. Pour simuler cet effet, on ajuste les variations concentrations- débits sur un modèle empirique de la forme $C=a \cdot Q^{-1}$. Le résultat obtenu n'est pas vraiment satisfaisant (bien que significatif d'un strict point de vue statistique, $R^{2}=0,67$ ). En effet, le modèle de dilution surestime systématiquement toutes les teneurs moyennes 
correspondant aux bas débits $\left(<0,3 \mathrm{~m}^{3} \cdot \mathrm{s}^{-1}\right)$. De fait, tout se passe comme si les teneurs de la rivière étaient " atténuées " aux bas débits. Pour rendre compte de ce phénomène nous testons divers modèles empiriques. Le meilleur ajustement est obtenu avec une équation de la forme : $P=$ a. $Q^{-1 / 2}$ soit $P$-tef $=$ $0,021+0,148 Q^{-1 / 2}\left(R^{2}=0,80 ; 82\right.$ valeurs ; $\left.F<0,0001\right)$ et $P$-total $=0,032+$ $0,157 \mathrm{Q}^{-1 / 2}\left(\mathrm{R}^{2}=0,79 ; 82\right.$ valeurs ; $\left.\mathrm{F}<0,0001\right)$. L'ajustement pour P-tef est présenté par la figure 5 a.
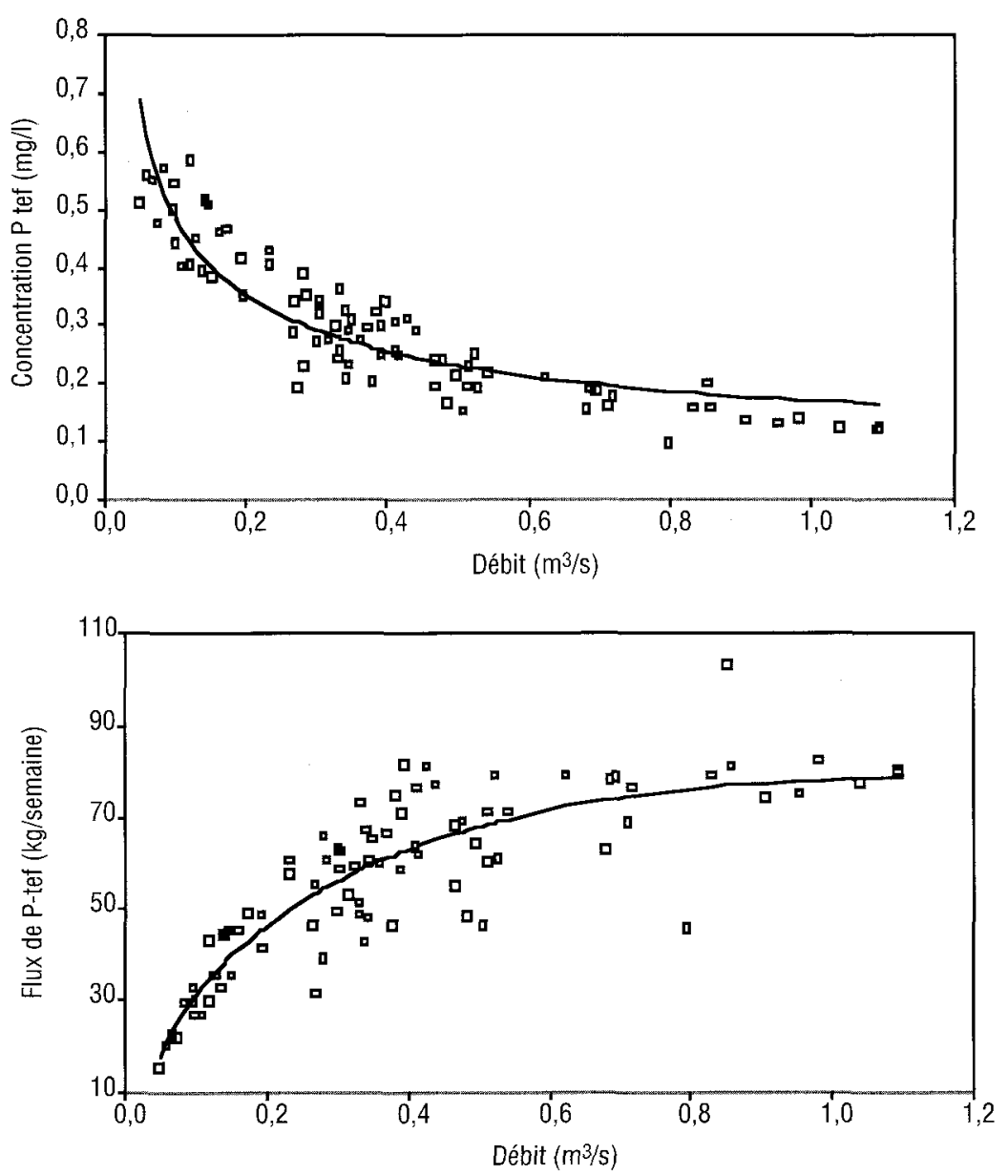

Figure 5a Relation concentration en phosphore total eau filtrée - débit, en période de tarissement.

Relation between dissolved $P$ concentrations and discharge during low flow periods.

Figure 5b Relation flux en phosphore - débit, lors des tarissements.

Relation between fluxes of dissolved $P$ ( $\mathrm{kg}$ per week) and discharge during low flow periods. 
La concentration en P-particulaire évolue différemment, indépendamment du débit, la moyenne restant basse $\left(<0,03 \mathrm{mgP} . \mathrm{I}^{-1}\right)$ et étroitement liée aux MES. Lors des semaines de tarissement, les MES et le P-particulaire proviennent principalement des rejets ponctuels (tableau 4). Les fortes charges en $P$ des MES (jusqu'à 2000 ppm) signent cette origine. Les conditions de débit (peu élevé, constant ou régulièrement décroissant) limitent la contribution des autres sources de MES possibles, abrasion du lit et des berges (les teneurs observées en P-part et MES dans les zones naturelles sont d'ailleurs très basses).

La traduction du comportement du P (P-total, P-tef ou ortho- $\mathrm{P}$ ) en terme de flux (figure $5 b$ ) met en relief d'autres phénomènes. Le flux hebdomadaire de $P$ à l'exutoire s'accroît progressivement, lorsque le débit moyen hebdomadaire varie du débit correspondant aux étiages sévères $\left(0,05 \mathrm{~m}^{3} . \mathrm{s}^{-1}\right)$ à un débit d'environ $0,8 \mathrm{~m}^{3} \cdot \mathrm{s}^{-1}$. Dans cette gamme de débit, les flux augmentent d'un facteur 4 (de 20 à $80 \mathrm{~kg} . P$ ). Au-delà de ce débit « limite » de $0,8 \mathrm{~m}^{3} \cdot \mathrm{s}^{-1}(\mathrm{QL}$ ), un plateau apparaît. Cette évolution du $P$ en fonction du débit lors des épisodes de tarissement, est à relier avec l'importance quantitative de sa rétention dans le réseau hydrographique.

\subsection{Mise en évidence de l'importance quantitative de la rétention du $\mathbf{P}$}

Pour mettre en évidence l'importance quantitative de la rétention du $P$ dans le réseau hydrographique, nous nous basons sur des bilans hebdomadaires en tarissement de $P$ (tableau 5). Nous choisissons : 1) de raisonner en $P$-total (puisque que le P-part comme le P-tef proviennent essentiellement des eaux usées en basses eaux) 2) d'utiliser des pas de temps proches ou supérieurs au mois, pour garantir au maximum la représentativité des rejets ponctuels moyens hebdomadaires minimum calculés. Les entrées dans le réseau sont constituées par les rejets ponctuels (constants en moyenne à l'échelle hebdomadaire) et par le «bruit de fond » dû aux apports des zones naturelles et agricoles. Le flux minimum moyen dû aux rejets est connu $(64 \mathrm{~kg} / \mathrm{semaine}$ par défaut). Le bruit de fond est estimé comme le produit entre les volumes d'eau exportés à l'exutoire et la teneur moyenne des eaux naturelles et agricoles (tableau 4). Le bilan entrée-sortie réalisé sur ces bases montre l'existence systématique d'un déficit important de $P$ à l'exutoire. Ce déficit (de 10 à $30 \mathrm{~kg}$ de P) est, à bas débit, supérieur aux flux exportés. En outre, il tend à varier en sens inverse du débit moyen. La rétention est donc un phénomène significatif vis-à-vis des transferts et potentiellement sensible aux conditions hydrologiques.

Si l'on généralise ces observations, l'augmentation du flux de $\mathrm{P}$ avec le débit de tarissement (figure $5 b$ ), résulte principalement d'une baisse progressive de la rétention. Le débit limite (QL) correspond alors à un débit suffisant pour transférer tout le phosphore rejeté dans le réseau hydrographique jusqu'à l'exutoire. En dessous de ce débit $\mathrm{QL}$, le $\mathrm{P}$ est retenu proportionnellement au débit, ce qui se traduit par un stockage dans le réseau hydrographique et un déficit dans les bilans entrées-sorties. Ce stockage expliquerait la distribution des teneurs en $\mathrm{P}$ des sédiments en étiage (tableau 3). 
Tableau 5 Premiers éléments pour un bilan de phosphore total à l'échelle de la semaine lors de 4 longues périodes d'étiage.

Table 5 Weekly mass balance of total phosphorus during 4 long low-flow periods.

\begin{tabular}{|c|c|c|c|c|c|}
\hline & & & & \multicolumn{2}{|c|}{$\begin{array}{l}\text { Flux moyen hebdomadaire de P-total } \\
\text { (kg par semaine) }\end{array}$} \\
\hline & & & & Entrée & Sortie à l'exutoire \\
\hline Période & $\begin{array}{l}\text { Durée } \\
\text { (jours) }\end{array}$ & $\begin{array}{l}\text { Débit moyen } \\
\left(\mathrm{m}^{3} \cdot \mathrm{s}^{-1}\right)\end{array}$ & $\begin{array}{l}\text { Bruit } \\
\text { de fond }\end{array}$ & $\begin{array}{l}\text { minimum } \\
\text { dúe } \\
\text { aux égouts }\end{array}$ & Moyenne \\
\hline $\begin{array}{l}05 / 08 \text { au } 16 / 09(1991) \\
13 / 08 \text { au } 24 / 09(1990) \\
15 / 02 \text { au } 22 / 03(1993) \\
20 / 01 \text { au } 10 / 02(1992)\end{array}$ & $\begin{array}{l}41 \\
41 \\
35 \\
21\end{array}$ & $\begin{array}{l}0,07 \\
0,12 \\
0,22 \\
0,29\end{array}$ & $\begin{array}{l}1,0 \\
1,3 \\
1,3 \\
1,1\end{array}$ & $\begin{array}{l}64 \\
64 \\
64 \\
64\end{array}$ & $\begin{array}{c}27,2 \\
34 \\
43,7 \\
53\end{array}$ \\
\hline
\end{tabular}

\subsection{Effets des caractéristiques hydrologiques antérieures}

La dispersion des points autour de la courbe flux - débit (figure $5 \mathrm{~b}$ ) ou concentrations-débits est importante, entre $0,3 \mathrm{~m}^{3} \cdot \mathrm{s}^{-1}$ et $0,6 \mathrm{~m}^{3} \cdot \mathrm{s}^{-1}$. Elle n'est pas liée à la durée du tarissement, comme en témoigne la relative stabilité des flux lors des étiages prolongés. Elle n'est pas non plus reliée statistiquement à la température de la rivière. Elle résulte certainement en partie d'aléas non évaluables (rejets accidentels et inévitables, écarts autour de la valeur moyenne des rejets hebdomadaires). Des phénomènes physiques semblent aussi intervenir : l'existence d'une rétention du P-total particulièrement élevée lors des semaines de tarissement succédant aux fortes crues, suggère un effet des antécédents hydrologiques.

Cette hypothèse d'un effet particulier des crues antérieures est testée en introduisant dans le modèle de régression précédemment établi $(C=f(Q))$ des variables qui cherchent à exprimer la capacité de ces crues à modifier l'état de la rivière et des sédiments et, au-delà, la capacité totale de fixation du P. Plusieurs modèles de régression sont testés; le modèle de régression multifactorielle retenu se présente sous la forme suivante:

$$
\text { P-tef }=0,049+0,14 \mathrm{Q}^{-1 / 2}-76^{\star} 10^{-6} \text { MES }
$$

avec : P-tef = concentration en $\mathrm{mg} \cdot \mathrm{f}^{-1} ; \mathrm{Q}=$ débit à l'exutoire des périodes de tarissement en $\mathrm{m}^{3} \cdot \mathrm{s}^{-1}$; MES = charge en matières en suspension exportées lors des crues antérieures, en tonne.

La relation globale obtenue est statistiquement significative avec un $R^{2}=$ 0,81 et probabilité $<0,0001$. L'introduction du paramètre MES est significative avec une probabilité $F=0,02$. La réduction de la dispersion des résidus se manifeste surtout dans la gamme des débits moyens $\left(0,2\right.$ à $\left.0,6 \mathrm{~m}^{3} / \mathrm{s}\right)$. Le même type de résultat est obtenu avec $P$-total, en concentration ou en flux.

La concentration et le flux du P-tef et du P-total lors des tarissements, dépendent donc principalement du débit de la période considérée et, secondairement, des caractéristiques hydrologiques des crues antérieures, qui s'expriment ici par la charge en MES. Ce paramètre peut être interprété comme une mesure du renouvellement des sédiments et des biofilms et de leur pouvoir fixateur, consécutif à de fortes crues. 


\section{5 - DISCUSSION}

\subsection{Conséquences des phénomènes observés en terme de bilan et des origines du phosphore}

\subsubsection{Mesure de la pollution ponctuelle}

À partir des résultats obtenus, il est possible de fournir une mesure de la pollution ponctuelle en $P$-total rejetée dans le réseau hydrographique, indépendante des ratios et des enquêtes (tableau 6). En effet, lors des semaines de tarissement présentant un débit moyen supérieur au débit limite (QL), les entrées dans le réseau hydrographique sont transférées intégralement jusqu'à l'exutoire. Or, le flux moyen hebdomadaire exporté à l'exutoire, soit de 90 à $110 \mathrm{~kg}$ de P-total équivaut, pour ces périodes, au total apporté par le bruit de fond et les rejets ponctuels. Pour la suite du calcul on retiendra une valeur moyenne de $93 \mathrm{~kg}$ de $\mathrm{P}$-total par semaine, moyenne arithmétique des flux enregistrés au-delà de QL. En déduisant le bruit de fond (estimé sur les bases explicitées précédemment) on peut donc déterminer la valeur moyenne hebdomadaire des rejets de pollution ponctuelle à $82 \mathrm{~kg}$, soit $4,2 \mathrm{t}$ de P-total par an. Ce rejet correspond à 4000 équivalents habitants environ et s'effectue pour l'essentiel sous des formes dissoutes (tableau 4).

Tableau 6 Calcul de la pollution ponctuelle à partir du bilan entrée-sortie lors des tarissements.

Table 6 Evaluation of point sources from the input-output balance during lowflow periods ( $k g$ total-P per week).

\begin{tabular}{lcccc}
\hline & $\begin{array}{c}\text { Sortie hebdomadaires } \\
(\mathbf{k g ~ P )}\end{array}$ & $\begin{array}{c}\text { Entrées hebdomadaires } \\
(\mathbf{k g ~ P )}\end{array}$ & & \\
\hline & $\begin{array}{c}\text { Flux maximum } \\
\text { exporté } \\
\text { en tarissement } \\
(\mathbf{1})\end{array}$ & $\begin{array}{c}\text { Flux dô } \\
\text { au bruit de fond } \\
(\mathbf{2})\end{array}$ & $\begin{array}{c}\text { Flux } \\
\text { de pollution } \\
\text { ponctuelle } \\
(\mathbf{3})=(\mathbf{1 - 2})\end{array}$ & $\begin{array}{c}\text { Flux } \\
\text { minimum } \\
\text { répertorié } \\
\text { par enquête }\end{array}$ \\
\hline Valeurs extrêmes & $90-110$ & $10-12$ & $80-95$ & - \\
Valeur moyenne & 93 & 11 & 82 & 64 \\
\hline
\end{tabular}

En comparant le résultat de ce calcul, avec le bilan réalisé sur la base d'enquêtes et de parcours de terrain il apparaît :

1) que $0,7 \mathrm{t}$ de $P$-total au moins, soit $30 \%$ des sources ponctuelles, n'avaient pas été prises en compte dans le bilan ( $X=0,7$ t dans figure 3$)$;

2) qu'au moins 3,1 t de P-total ( $Y$ voir figure 3) rejeté dans le bassin par les égouts domestiques, ne se retrouvent pas à l'exutoire!

Nous penscns que les sources ponctuelles non identifiées correspondent en grande partie aux 70 tuyaux recensés comme rejetant des eaux usées dans le réseau hydrographique. Par ailleurs, le phosphore manquant est fixée par les sous-sols et les sols du bassin. Les stocks ainsi créés se cumulent au fil des années au rythme théorique de $1,2 \mathrm{~kg} / \mathrm{ha} / \mathrm{an}$, qui s'ajoutent aux $15 \mathrm{~kg} / \mathrm{ha} / \mathrm{an}$ dus aux excédents moyens des bilans entrées-sorties des sols agricoles de la 
région. La question qui se pose désormais est celle de la stabilité de ces stocks et de leur devenir à long terme.

\subsubsection{Prise en compte du stock de phosphore du réseau hydrographíque dans les bilans de bassin}

L'existence d'une rétention de phosphore pour des débits de tarissement inférieur au débit limite aboutit à la création de stocks importants dans le réseau hydrographique. Ainsi, durant les 6 semaines consécutives d'étiage de l'été 1990 (tableau 5) les exportations mesurées (204 kg de P-total) ne représentent que $40 \%$ du total des entrées hebdomadaires dans le réseau (rejets ponctuels moyens $82 \mathrm{kgP}$-total, et bruit de fond $7,8 \mathrm{~kg}$ de P-total). La quantité retenue est donc d'environ $320 \mathrm{~kg}$ de $\mathrm{P}$-total, ce qui équivaut à $7 \%$ des rejets ponctuels annuels ou encore aux exportations d'une semaine de forte crue ( $Q$ moy. = 1 à $3 \mathrm{~m}^{3} \cdot \mathrm{s}^{-1}$ ).

Dans le bassin voisin du Redon, le stock maximum enregistré, en 4 ans d'étude, correspondait au quart du flux annuel de P-total, soit une valeur de l'ordre de grandeur des flux diffus (PILLEBOUE, 1987a). Les quantités de P-total stockées en tarissement sont donc loin d'être négligeables; elles doivent être prises en compte dans les bilans pour éviter d'attribuer les variations de stocks au terme du bilan souvent connu par différence, c'est-à-dire à la pollution diffuse. Dans la pratique, il s'agit simplement de commencer et clore les bilans juste après de très fortes crues susceptibles d'évacuer l'ensemble des stocks et de renouveler le pouvoir fixateur de la rivière. En respectant cette règle, il est possible de réaliser des bilans sur des périodes présentant des variations de stock nulles. Cette précaution peut probablement s'étendre aux bassins ne comprenant que des sources diffuses (JORDAN-MEILLE, 1998). Empiriquement, nous avons fixé le débit moyen de ces fortes crues à 5 fois au moins le débit limite.

Sur ces bases, pour établir le bilan du Foron, nous avons considéré 4 périodes d'une durée variant de 6 à 13 mois, chaque période étant encadrée par de fortes crues (tableau 7). Le terme du bilan correspondant à la différence relative entrées ponctuelles-sorties à l'exutoire, varie énormément (de 9 à $24 \%$ du P-total exporté) mais sans relation directe avec le volume écoulé. Ce terme intègre les diverses pollutions diffuses (agricoles et urbaines) ainsi que le bruit de fond. Si on se réfère à la synthèse bibliographique de PILLEBOUE (1987a) et aux estimations locales, JORDAN-MEILLE (1998), les apports diffus liés au ruissellement urbain contribuerait au maximum à $1,5 \mathrm{~kg}$ de $P$ total/ha/an, soit environ $100 \mathrm{~kg} / \mathrm{an}$ pour tout le bassin. De même les pertes provenant des zones naturelles contribueraient pour moins de $0,1 \mathrm{~kg}$ de Ptotal/ha/an, soit $250 \mathrm{~kg} / \mathrm{an}$ pour le bassin. Compte tenu de ces contributions, les pertes diffuses des zones agricoles (surface environ 1800 ha) peuvent être évaluées à $600 \mathrm{~kg} / \mathrm{an}$ de P-total, soit un flux spécifique de $0,3 \mathrm{~kg}$ de $\mathrm{P}$ total/ha/an. Cette estimation est très proche de celle obtenue sur dans le bassin du Redon dont la zone agricole est également dominée par des herbages. Elle est cohérente avec les données de la bibliographie qui situent les pertes diffuses en $P$ total des herbages non drainées entre 0,1 et $0,5 \mathrm{~kg} / \mathrm{ha} / \mathrm{an}$ (DORIOZ, 1996). 
Tableau 7 Bilan des entrées de phosphore total $(\mathrm{kg})$ pendant plusieurs périodes hydrologiques débutant et se terminant par de fortes crues.

Table $7 \quad$ Phosphorus balance $(\mathrm{kg})$ during long hydrological periods beginning and ending with a strong high flow event.

\begin{tabular}{|c|c|c|c|c|c|c|}
\hline Dates & $\begin{array}{l}\text { Durée } \\
\text { (jour) }\end{array}$ & $\begin{array}{c}\text { Sorties de P } \\
\text { à l'exutoire } \\
\text { (mesurées) } \\
(\mathrm{kg})\end{array}$ & $\begin{array}{c}\text { Entrée de P } \\
\text { ponctuelle } \\
\text { (calculées) } \\
(\mathrm{kg})\end{array}$ & $\begin{array}{l}\text { Différences } \\
\text { sorties-entrées } \\
(\mathrm{kg})\end{array}$ & $\begin{array}{c}\text { Contributions } \\
\text { relatives } \\
\text { des pollutions } \\
\text { diffuses } \\
(\%)\end{array}$ & $\begin{array}{c}\text { Volume } \\
\text { écoulé } \\
\left(10^{3} \mathrm{~m}^{3}\right. \\
\text { par jour })\end{array}$ \\
\hline $\begin{array}{c}16 / 02 / 90 \text { au } \\
09 / 09 / 90\end{array}$ & 205 & 1919 & 1466 & 454 & 24 & 58,5 \\
\hline $\begin{array}{c}09 / 09 / 90 \text { au } \\
23 / 03 / 91\end{array}$ & 195 & 4063 & 3686 & 377 & 9 & 66,6 \\
\hline $\begin{array}{c}23 / 03 / 91 \text { au } \\
14 / 11 / 91\end{array}$ & 235 & 3730 & 3021 & 709 & 19 & 44,7 \\
\hline $\begin{array}{c}14 / 11 / 91 \text { au } \\
08 / 12 / 92\end{array}$ & 389 & 5698 & 5079 & 620 & 11 & 63,7 \\
\hline Total & 1024 & 15410 & 13251 & 2159 & 14 & 233,5 \\
\hline
\end{tabular}

\subsection{La relation concentration-débit en tarissement, indicateur d'état d'un bassin?}

En tarissement, les apports de $\mathrm{P}$ sont en globalement constants à l'échelle hebdomadaire ; c'est leur taux de transmission à l'exutoire qui varie avec le débit. De ce fait, la relation concentration moyenne hebdomadaire - débit moyen hebdomadaire n'est pas une simple loi de dilution et l'écart observé par rapport à la loi de dilution traduit la rétention du $P$ dans le réseau et la rivière (figure 6).

Les informations fournies par la courbe concentration-débit sont donc doubles : 1) l'écart avec la courbe de dilution décrit le pouvoir de rétention global du réseau hydrographique et sa variation selon le débit 2) les teneurs observées au niveau du débit limite sont proportionnelles au total des rejets ponctuels. Ainsi interprétée, cette relation pourrait constituer un outil pour évaluer l'efficacité d'interventions réalisées sur le bassin versant : la baisse de la teneur au débit limite indique une diminution proportionnelle des apports, alors que les changements de forme de la courbe ou de débit limite révèlent des modifications concernant les modalités de la rétention. Une approche similaire peut être envisagée pour les nitrates (PILLEBOUE, 1987a). Une comparaison avec la dynamique d'ions solubles conservatifs comme $\mathrm{Cl}^{-}(\mathrm{ABECK}, 2000)$, pourrait constituer un traçage complémentaire. 


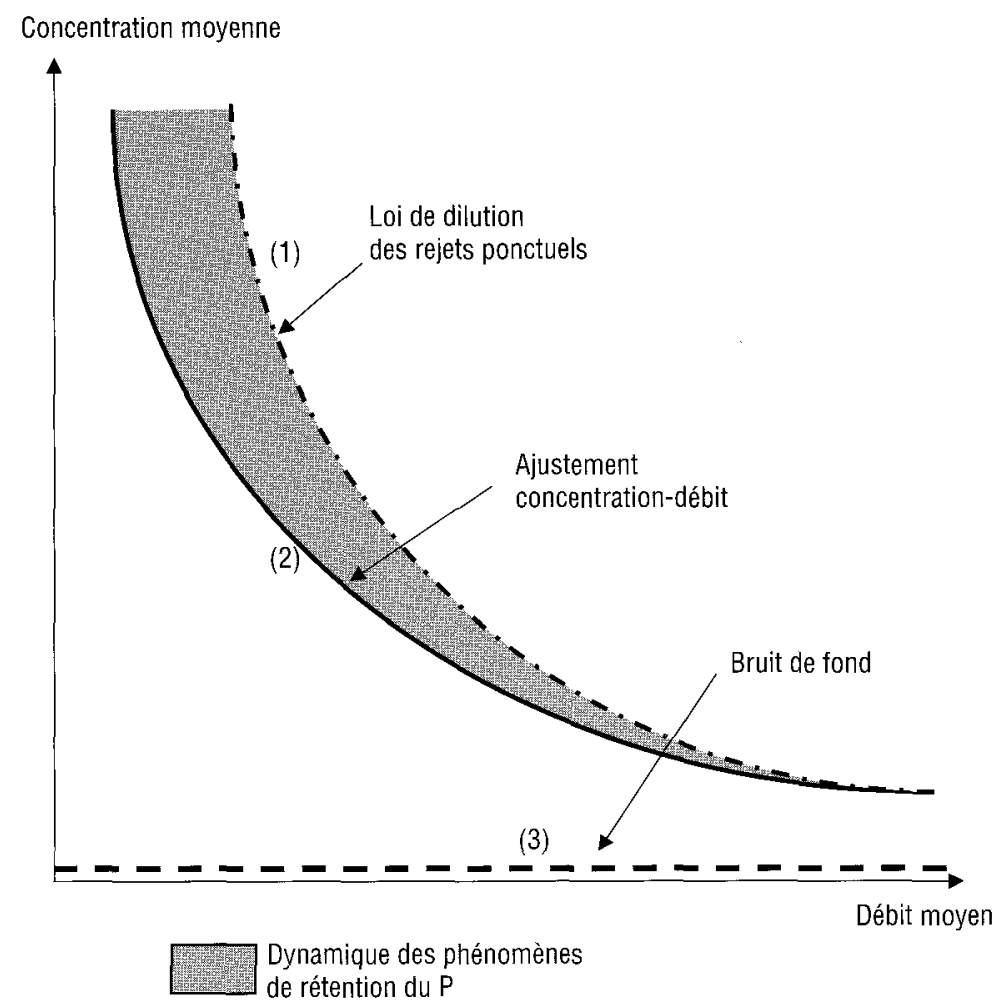

Figure 6 Interprétation de la relation concentration-débit, en période de tarissement.

Theoretical relation between concentration and discharge during low flow periods.

\subsection{Mécanismes de la rétention du phosphore}

Nos résultats confirment que la rétention joue un rôle central dans la dynamique du phosphore à l'échelle des bassins versants (DORIOZ et al., 1989 ; SVENDSEN et al., 1995 ; OWENS et WALLING, 2002). L'intensité de ce phénomène est très liée à l'hydrologie et à la concentration en phosphore dissous (CASSELL, 1995). Dans le Foron, il est aussi lié à l'état de la rivière : plus la crue antérieure est puissante et chargée en suspensions, plus le renouvellement des sédiments de la rivière et l'abrasion du périphyton est complet et plus, en conséquence, la capacité de rétention est élevée.

Dans les rivières torrentielles et dépourvues de macrophytes, comme celle du bassin lémanique, la rétention met en jeu l'enrichissement en $P$ des sédiments, des biofilms et des suspensions, au contact d'une eau fortement chargée en $P$ dissous. L'enrichissement des sédiments s'accompagne probablement d'une augmentation de la biodisponibilité potentielle du P-particulaire, comme le suggère la relation entre teneur en $\mathrm{P}$ - total et en $\mathrm{P}$-Olsen des sédiments. Le relargage du $P$ retenu est réalisé lors des crues. Dans d'autres 
rivières, plus calmes, ce sont les macrophytes qui jouent un rôle clef (SVENDSEN et KRONVANG, 1993). Le relargage du $P$ retenu s'opère alors pour l'essentiel à l'automne.

La rétention, affecte principalement le $\mathrm{P}$-dissous. Elle résulte :

1) d'un piégeage direct, soit par fixation biologique (MEYER et LIKENS, 1979), au niveau des végétaux périphyton; soit par fixation physico-chimique sur les sédiments ;

2) d'un simple piégeage passif dans l'eau interstitielle des sédiments ;

3) de mécanismes d'insolubilisation réalisant une transformation des formes dissoutes en $P$ particulaire, soit par précipitation (GOLTERMAN, 1976), soit par adsorption sur les suspensions (HARM et al., 1978; WANG, 1974), suivi d'une sédimentation, elle-même favorisée par la présence de macrophytes ou d'obstacles ou d'un lit de galets (CASSEL, 1995) ; le P-particulaire rejeté avec les eaux usées ou les colloïdes après coagulation, subissent la même sédimentation.

Tous ces processus co-existent dans les rivières mais il est bien difficile, à partir des données disponibles, de déterminer si l'un d'entre eux a un rôle prépondérant. En fait, les divers mécanismes d'insolubilisation semblent se combiner avec la sédimentation, pour aboutir à la rétention du $P$.

\subsection{Transformations de la spéciation associée à la rétention et devenir du P stocké}

De nombreuses études montrent que le P-total accumulé dans le réseau pendant les basses eaux est remis en circulation lors des crues, en totalité ou en partie, selon la puissance de la crue et les caractéristiques de la rivière (HARMS et al., 1978 ; DORIOZ et al., 1989 ; SVENDSEN et al., 1995 ; CANN, 1995). Dans le Foron, comme dans le Redon, les crues succédant à des périodes de rétention ont des caractéristiques spécifiques: flux et concentrations élevés, notamment en $\mathrm{P}$-tef et Ortho-P, fortes teneurs en $\mathrm{P}$ des suspensions, rapport N/P... (DORIOZ et al., 1989 ; DORIOZ et al., 1998a). Ceci traduit les conditions de re-mobilisation du P-total stocké : re-suspension de particules fortement enrichies en $\mathrm{P}$ et libération de formes dissoutes, notamment à partir d'eau interstitielle. Les processus impliqués dans la fixation du $P$ sur les particules étant partiellement réversibles, il est probable que la re-suspension de sédiments enrichis aboutisse aussi à re-solubiliser une partie du $\mathrm{P}$ fixé lors des tarissements. Ce phénomène, en théorie, est d'autant plus intense que le $P$ particulaire des sédiments comprend une fraction élevée de formes labiles, ce qui est la règle en étiage dans presque tout le réseau hydrographique étudié.

L'étude du bilan entrée-sortie du P-tef dans le Foron soutient l'hypothèse que les transformations de spéciation $\mathrm{P}$-tef $\leftrightarrow P$-part sont quantitativement significatives. Les termes de ce bilan pour les 4 années du suivi sont les suivants:

1) les entrées dans la rivière, soit 4,6 t.P-tef/an, représentent la somme des apports par les rejets ponctuels soit $4 \mathrm{t}$ (86 kg.P-total/semaine, composé de $90 \%$ de $P$-tef), du bruit de fond soit 0,2 t/an (volume écoulé hors périodes de crue * concentration des nappes en P-tef), de la fraction P-tef des apports diffus urbains et agricoles, soit 0,4 t.P-total/an (diffus $=0,8 \mathrm{t}$ /an de P-total avec, hypothèse haute, la moitié sous forme dissoute selon les estimations locales de PILLEBoue, 1987a et JoRdAN-MEILLE, 1998), 
2) les sorties moyennes soit 3,6 t/an de P-tef mesuré à l'exutoire.

Selon ces estimations, il existe donc un déficit des sorties de P-tef d'un peu moins d'une tonne. Le même type de résultats est obtenu par SVENSEN et al. (1995). P-total étant conservatif, ceci suppose une transformation P-tef $\rightarrow$ P-Particulaire correspondant à environ un tiers du P-particulaire exporté annuellement.

La transformation de P-tef, provenant principalement des égouts, en formes particulaires, diminue probablement la biodisponibilité potentielle du P-total transféré, car le P-particulaire est systématiquement moins biodisponible que les formes dissoutes (SONZOGNI et al., 1981). Cependant, le P-particulaire ainsi généré présente (DORIOZ et al., 1998b) une biodisponibilité largement supérieure à celle du phosphore lié aux sédiments de n'importe quelle autre origine.

Par ailleurs, la transformation P-tef $\rightarrow$ P-part. induit un retard à l'exportation qui pourrait s'avérer bénéfique dans la mesure où le transfert vers le lac s'effectue lors de périodes de faible activité biologique de celui-ci. Au-delà de ces considérations théoriques, aucune donnée actuelle ne permet d'évaluer avec précision l'éventuelle baisse des impacts due à ces transformations.

\section{6 - CONCLUSIONS}

Le transfert dans le réseau hydrographique de phosphore provenant de sources ponctuelles se caractérise par une alternance de phases de rétention et de transport. Cette dynamique a deux conséquences.

- Il apparaît un retard à l'exportation vers les milieux récepteurs sensibles et des modifications de spéciation liée à une insolubilisation d'une partie du $P$ dissous. La baisse d'impact potentiel qui résulte de ces phénomènes reste à quantifier.

- Le taux de transfert des rejets ponctuels de $P$ s'accroît avec le débit ce qui atténue les effets de la dilution. En tarissement, cette double dynamique détermine la forme de la relation concentration en $\mathrm{P}$ - débit.

Les relations concentration de phosphore-débit en tarissement, représentent un outil opérationnel pour évaluer l'intensité et les facteurs contrôlant la rétention du $\mathrm{P}$ dans le réseau. Elles pourraient aussi être utilisées comme indicateurs dans les suivis à long terme, ou pour estimer globalement les rejets ponctuels. Cette dernière application fournit au moins dans certains types de rivière, une estimation dans une fourchette de valeur de 20 à $30 \%$. Elle est donc susceptible de compléter et voir de remplacer les évaluations issues d'inventaires et de ratios. 


\section{REMERCIEMENTS}

Les auteurs remercient J.-Y. VANSTEELANT pour sa relecture attentive, A. FERHI (Paris VI) pour ses conseils en matière d'hydrologie et le SIVOM du Bas-Chablais pour son aide sur le terrain.

\section{RÉFÉRENCES BIBLIOGRAPHIQUES}

ALBECK E., 2000. Identification of different sources of chlorides in streams by regression analysis using chloridedischarge relationship. Water Env. Res., 71, 7, 1310-1319.

BALLAND P., 1989. Origine domestique des apports en phosphore. AIDEC. Colloque phosphore, cahier 28, tome 1, 51-80.

BARROIN G., 1992. La pollution des eaux stagnantes par les phosphates. Contreverses et décisions politiques. Environnement, sciences et politiques. Tome II. Germes, cahiers 14.

BEHRENDT $H$. et BACHOR A., 1998. Point and diffuse load of nutrients to the baltic sea by rivers basins of North Germany. Wat. Sci. Tech., 38, 10, 147-155.

BOUVIER O. 2003. Bilan et dynamique du phosphore l'échelle d'un bassin versant. Aspects méthodologiques. Applications à un sous bassin versant du lac Léman. DESS «Ingénierie de l'eau - Mesures et Méthodes "Bordeaux III. $72 \mathrm{p}$.

CANN C., 1995. Flux de nutriments d'origine agricole vers la rade de Brest. Ingénieries EAT, 37-44.

CASSELL E.A., 1995. The dynamic streamphosphorus model. A dynamic simulation model for descriving $P$ cycling, transport and storage in streams. Final report, School of Natural Ressources, Univ. of Vermont, Burlington, VT, USA, $60 \mathrm{p}$.

CASTANY G., 1962. Études des eaux souterraines. Cours à la Faculté des Sciences de Paris.

CIPEL, 1991. Le Léman demain, plan d'action. Commission Internationale pour la
Protection des Eaux du Léman contre la pollution. Lausanne; $20 \mathrm{p}$.

DORIOZ J.M., 1996. Transferts de phosphore des bassins versants agricoles aux eaux de surface : ordre de grandeur, mécanismes, facteurs de risque. Maîtrise. Colloque INRA $50^{\circ}$ anniversaire. Sécheresse, pollution, inondation, érosion. Poitiers, $15 p$.

DORIOZ J.M., PILLEBOUE E., FERHI A., 1989. Dynamique du phosphore dans les bassins versants: importance des phénomènes de rétention dans les sédiments. Wat. Res.,. 23, 2, 147-158.

DORIOZ J.M., PILLEBOUE E., ORAND A., 1991. Prélèvement et échantillonnage dans les petits bassins versants ruraux. Revue des Sciences de l'eau 4, 211-238.

DORIOZ J.M., CASSEL A., ORAND A., EISENMAN K., 1998a. Phosphorus storage, transport and export dynamics in the Foron river watershed. Hydrol. Processes, 12, 285-309.

DORIOZ J.M., PELLETIER J., BENOIT P., 1998 b. Variations des propriétés physico-chimiques et de la biodisponibilité potentielle du phosphore particulaire selon l'origine des sédiments dans un bassin versant. Water Res., 32, 2, 275286.

DORIOZ J.M., TRÉVISAN D., 2002. Transferts de Phosphore des bassins versants agricoles vers les eaux de surface : l'expérience du bassin Lémanique (France) et sa portée générale. IRDA Québec. Agrosols, 12, 2, 85-97.

FRERE M.H., WOOTHISER D.A., CARD J.H., WISCHMEIER W.H., 1978. Control of non point water pollution from agricul- 
ture: some concepts. J. Soil Water Conserv., 32, 6, 200-264.

GOLTERMAN H. [ Ed.], 1976. Interactions between sediments and fresh water. Proc. Int. Symp. Amsterdam.

HAMID S., DRAY M., FERHI A., DORIOZ J.M., FONTES J.C., 1989. Étude des transferts d'eau à l'intérieur d'une formation morainique du Léman. $J$. of Hydr., 109, 369-385.

HARM L., VIDAL P.H., MC.DERMOTT T.G., 1978. Phosphorus interactions with streambed sediments. J. Envir. Eng. Div., 104, EE2, 271-288.

JORDAN - MEILLE L., 1998. Modes de transfert du phosphore d'origine diffuse dans un petit bassin agricole rural lémanique. Thèse Dép. de Génie rural. EPFL (Ch), n ${ }^{\circ} 1775,289 \mathrm{p}$.

JORDAN - MEILLE L, DORIOZ J.M. , WANG D., 1998. Analysis of the export of diffuse phosphorus from a small rural watershed. Agronomie, 18, 5-26.

KRONVANG B., 1992. The export of particulate matter, particulate phosphorus and dissolved phosphorus from two agricultural river basin : implications on estimating the non point phosphorus load. Water Res., 26, 10, 1347-1358.

MEALS D., BUDOLF F., 1994. Lake Champlain non point source. Pollution assessment tech. Report 6A Lake Champlain Manag. Conference. $60 \mathrm{p}$.

MEALS D.W. ,LEVINE S., WANG D., CASSEL E., DRAKE J.C., PELTON D.K., GALARNEAU H.M., BROWN A.B., 1999. Retention of spike additions of soluble phosphorus in a norten eutrophic stream. J. N. Am.Benthol. Soc., 18, 2, 185-198.

MENZEL O.H., CORVIN N., 1965. The measurement of total phosphorus in seawater based on, the liberation of organically bound fraction by persulfate oxydation. Limnol. Ocean., 10, 2, 280-292.

MEYER J.L., LIKENS G.E., 1979. Transport and transformation of phosphorus in a forest stream ecosystem. Ecology, 60, 1255-1269.

MURPHY J., RIPLEY J.P., 1968. A modified single solution method for the determination of phosphate in natural waters. Anal Chemo. Acta., 27, 21-36.
NOVOTNY V., OLEN H., 1994. Water quality: prevention identification and management of diffuse pollution. Van nostrand Reynold, NewYork.

OLSEN S.R., COLLE C.V., WATANABE, DEAN L.A, 1955. Estimation of available phosphorus in soils by extraction with sodium bicarbonate. USDA Washington, $\mathrm{n}^{\circ} 939$.

OWENS P.N., WALLING D.E., 2002. The phosphorus content of fluvial sediment in rural and industrial river basins. Water Res., 36, 685-701.

PILLEBOUE E., 1987a. Origines, bilans, mécanismes de transfert du phosphore et de l'azote d'un bassin versant à un lac. Thèse Doct. Univ. Paris 6, $250 \mathrm{p}$.

PILLEBOUE E., 1987b. Estimation de la pollution diffuse dans un bassin versant hétérogène. Sciences de l'eau, 6, 1, 49-65.

PROBST J.L., 1983. Hydrologie du bassin de la Garonne. Modèle de mélange. Exportation de phosphate et nitrate. Thèse Doct. Univ. P. Sabatier Toulouse, $168 \mathrm{p}$.

PROBST J.L., KATTAN Z., ETCHANCHU D, 1985. Exportation des nitrates sur 4 bassins versants français agricoles. Congrès Nitrates dans les eaux. 22-24 octobre 1985. Paris.

RYDING S.O., RAST W., 1994. Le contrôle de l'eutrophisation des lacs et des réservoirs. Masson. Unesco Paris, 294 p.

SAUNDERS W.M.H., WILLIAMS E.G., 1955. Observation on the determination of total organic phosphorus in soils. $J$ of Soil Science, 6, 12, 254-262

SHARPLEY A.N., DANIEL T.C., EDWARDS D.R., 1993. Phophorus movement in the landscape. J. Prod. Agric., 6, 492-500.

SONZOGNI W.C., AMSTRONG D.E., LOGAN T.J., 1981. Bioavailability of phosphorus inputs to lakes: significance to management. Great lakes Env. Planning study, $\mathrm{n}^{\circ}$ 40. Great lakes basin commission, Ann Arbor, MI.

SVENDSEN L.M., KRONVANG B., 1993. Retention of nitrogen and phosphorus in a Danish lowland river system: implications for the export from the watershed. Hydrobiologia, 251, 123-135.

SVENDSEN L.M., KRONVANG B., KRISTENSEN P., GRAESBOL P., 1995. Dynamics 
of phosphorus compounds in a lowland river system: Importance of retention and non point sources. Hydro. Processes, 9, 119-142.

UUSI-KAMPA J.,TURTOLA E., HARTIKAINEN H., YLARANTA T., 1997. The interactions of buffer zones and phosphorus runoff. In Buffer zones edited by NE. Haycock, TP. Burt and G. Pinay.

VANSTEELANT J.Y., TREVISAN D., PERRON L., DORIOZ J.M., ROYBIN D., 1997. Conditions d'apparition du ruissellement dans les cultures annuelles de la région lémanique, relation avec le fonctionnement des exploitations agricoles. Agronomie, 17, 17-34.
VIAL, 1976. Études géologiques et hydrogéologiques de la région de Thonon. Thèse Doct. Univ. Grenoble, $170 \mathrm{p}$.

VOLLENWEIDER R., 1968. Scientific fundamentals of the eutrophication of lakes and flowing waters. Tech. Report OECD, Paris, $150 \mathrm{p}$.

WANG W.C., 1974. Absorption of phosphate by river particulate matter. Water Res. Bull., 10, 4, 662-671.

WILLIAMS J.D.H., SHEAR H., THOMAS R.L., 1980. Availabity to scenedesmus quadicanda of different form of phosphorus in sedimentary materials from great lakes. Limnol. Oceanogr., 25, 1-11. 